\title{
Correction: BiKEGG: a COBRA toolbox extension for bridging the BiGG and KEGG databases
}

Cite this: Mol. BioSyst., 2016 , 12,3743

DOI: $10.1039 / \mathrm{c} 6 \mathrm{mb} 90040 \mathrm{~b}$

www.rsc.org/molecularbiosystems

\author{
Oveis Jamialahmadi, Ehsan Motamedian and Sameereh Hashemi-Najafabadi*
}

Correction for 'BiKEGG: a COBRA toolbox extension for bridging the BiGG and KEGG databases' by Oveis Jamialahmadi et al., Mol. BioSyst., 2016, DOI: 10.1039/c6mb00532b.

Web links to download the BiKEGG toolbox were missing from this article. Apart from the supplementary material included with the paper for downloading the BiKEGG toolbox, the authors would like to add additional resources for downloading the BiKEGG toolbox. The toolbox is hosted on GitHub (https://github.com/Ojami/BiKEGG), and is available at https://bikegg.github.io/, for both bug tracking and community contribution. Furthermore, the BiKEGG project can be found on the 'Systems Biology and Metabolic Engineering Laboratory' website at http://sbme.modares.ac.ir/.

The Royal Society of Chemistry apologises for these errors and any consequent inconvenience to authors and readers. 